\title{
Apical Variant of Hypertrophic Cardiomyopathy Presenting as Recurrent Syncope and Wide Complex Tachycardia
}

\author{
Navaraj Paudel*, V.M. Alurkar, G.S. Jha, Ramchandra Kafle, Subash Sapkota, Niraj Dhakal
}

Department of Cardiology, Manipal Teaching Hospital, Pokhara, Nepal.

DOI Name

http://dx.doi.org/10.3126/jaim.v6i2.18539 Keywords

Apical hypertrophic cardiomyopathy, Syncope, Wide complex tachycardia

\section{Citation}

Navaraj Paudel, V. M. Alurkar, G. S. Jha, et al. Apical variant of hypertrophic cardiomyopathy presenting as recurrent syncope and wide complex tachycardia. Journal of Advances in Internal Medicine 2017;06(02):32-34.

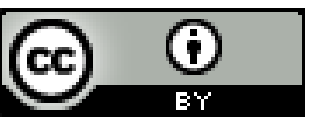

This work is licensed under a Creative Commons Attribution 3.0 Unported License.

\begin{abstract}
Apical hypertrophic cardiomyopathy $(\mathrm{AHC})$ is an uncommon variant of hypertrophic cardiomyopathy usually found as an incidental finding during routine echocardiography. It was first described by Sakamoto in 1976 in Japanese patients. Echocardiography is still considered as the best screening tool to assess AHC although MRI is considered as the gold standard for confirmation. In this case, we present a 21-year-old man with AHC who presented with recurrent brief syncopal episodes which were ignored by the patient until he was brought to us with prolonged syncope and wide complex tachycardia/ posterior fascicular ventricular tachycardia. We observed the classical findings in the plain twelve lead electrocardiograms done after cardioversion. It is always wise to keep special attention to evaluate hypertrophic cardiomyopathy (HCM) in young patients presenting with syncope.
\end{abstract}

\section{INTRODUCTION}

The most common cardiomyopathy is hypertrophic cardiomyopathy (HCM) is also the most common hereditary cardiac disease. The prevalence of HCM is as high as 1 in 5001000 live births in various parts of the world. ${ }^{1}$ There are multiple variants of HCM. A form of HCM characterized by localized hypertrophy of left ventricular apex is considered as Apical hypertrophic cardiomyopathy (AHC). ${ }^{2}$ Sakamoto et al. ${ }^{3}$ was the first to report AHC in Japan and subsequently by Yamaguchi et al. ${ }^{4}$ gave the full description. It is more common in Japan representing $25 \%$ of patients with $\mathrm{HCM}$, whereas, in nonAsian patients, the incidence of $\mathrm{AHC}$ is $1-2 \% .{ }^{5} \mathrm{AHC}$ has typical findings on electrocardiography (ECG), echocardiography and ventriculography. Giant T wave negativity which is defined as a voltage of negative $T$ wave $\geq 1 \mathrm{mV}$ ( $\geq 10 \mathrm{~mm}$ ) in any of the leads and high $\mathrm{R}$ wave voltage is the typical ECG picture in AHC. The ECG changes with symptoms associated with AHC often mimic acute coronary syndromes (ACS). ${ }^{6}$ Left ventriculography shows end-diastolic LV cavity configuration resembling an 'ace of spades'. Transtorasic doppler echocardiography often demonstrates the mid-ventricular obstruction. ${ }^{7}$ AHC is generally considered as having a benign prognosis, one-third of the patients may have severe complications such as myocardial infarction, atrial fibrillation and stroke. ${ }^{7}$ Supraventricular tachycardia is also seen in patients with $\mathrm{HCM} .{ }^{8}$ Ventricular tachycardia and sudden death are rare in cases with AHC. ${ }^{9,10}$ Here we report a case of apical hypertropic cardiomyopathy with symptoms with wide complex tachycardia. The ECG findings after cardioversion mimic ischemic heart disease.

\section{CASE:}

A 21 years old male athletic, an army recruit under training was having history of brief episodes of loss of consciousness since 3-4 years. The episodes were usually following heavy exercises. He didn't seek medical advice until he was brought for prolonged loss of consciousness by the family. Upon arrival in emergency, he was semiconscious, BP:76/50 $\mathrm{mmHg}$ with feeble pulse at the rate of $150 / \mathrm{min}$. ECG showed heart rate of $160 / \mathrm{min}$, extreme axis with positive $\mathrm{R}$ in aVR, RBBB pattern in V1, fusion and captured beats observed in lead III (figure 1)

\footnotetext{
* Corresponding author

Navaraj Paudel,

Manipal College of Medical Sciences

Manipal Teaching Hospital

Phulbari-11, Pokhara, Kaski, Nepal.

Email:drnavarajmd@gmail.com
} 
suggestive of VT (possibly posterior fascicular).

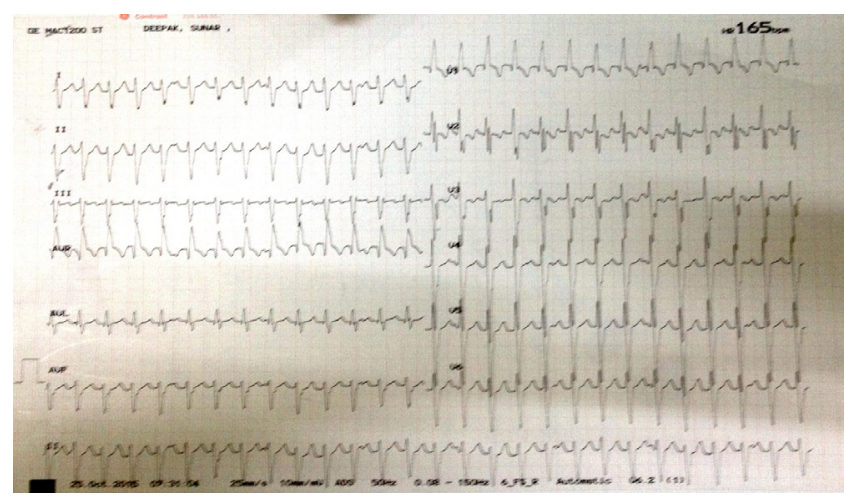

Figure 1: ECG of the patient during arrival in ER.

Immediately a DC cardioversion with 200 joules was done. His ECG was re-evaluated following cardioversion which showed normal axis, left ventricular hypertrophy (voltage criteria), deep T inversion in V4-V6, II, III and aVF were seen. Small notch at the later part of $\mathrm{R}$ wave seen. (figure 2).

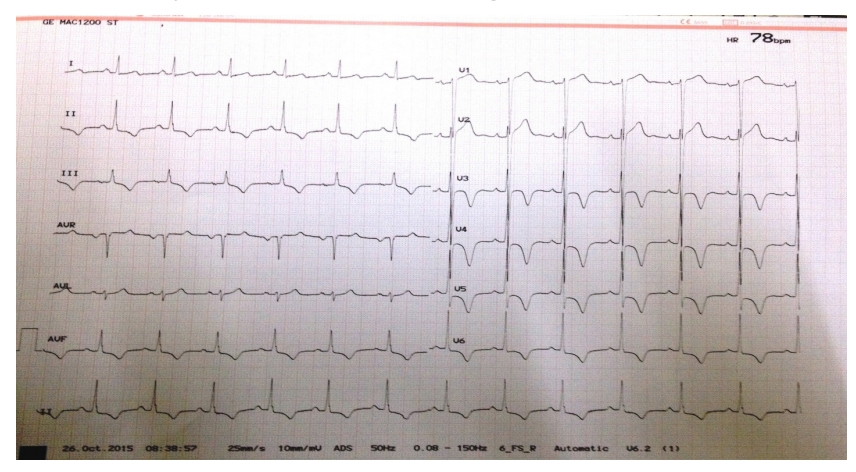

Figure 2: ECG of the patient following cardioversion.

2-D transthorasic echocardiography showed asymmetric left ventricular hypertrophy (LVH), confined predominantly to the LV apex with an apical wall thickness $16 \mathrm{~mm}$ and a ratio of maximal apical to posterior wall thickness 1.6 (figure 3). Chet X-ray was normal (figure 4).

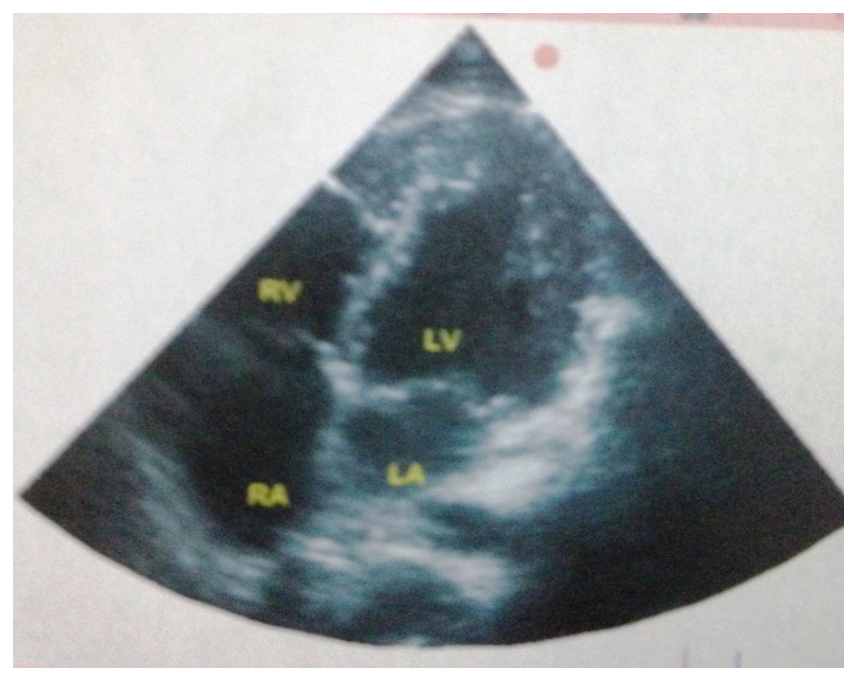

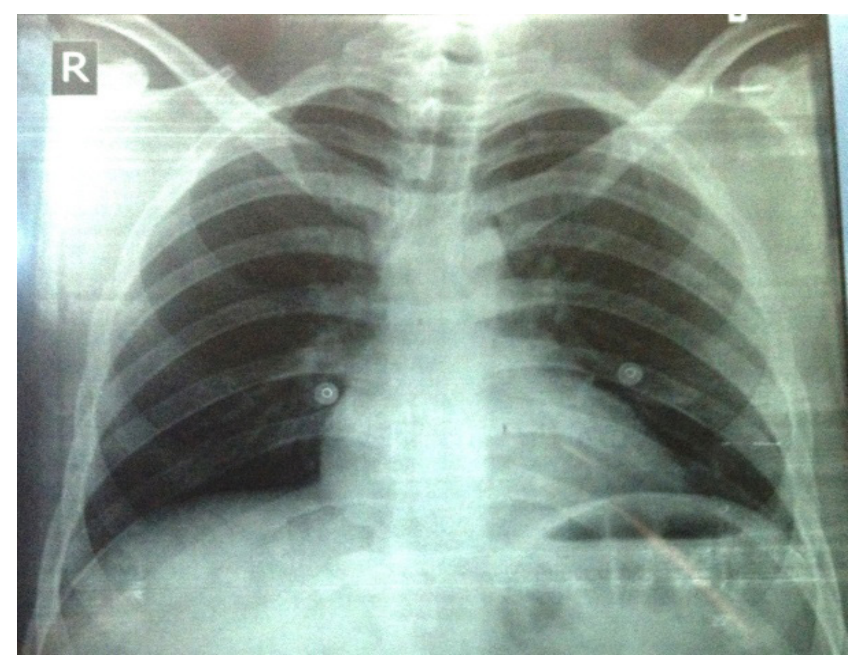

Figure 3 (left) shows echocardiography of the patient showing asymmetric thickening in LV apex (arrow). Figure 4 (right) is the chest X-ray of the patient.

Screening echocardiography of two of his siblings and parents was done which was absolutely normal. He was kept on beta blocker. He was advised for electrophysiological study and radiofrequency ablation.

\section{DISCUSSION:}

AHC has a significant proportion of the overall HCM. In general, AHC has a benign prognosis. Diagnosis is based on typical findings on ECG, echocardiography and ventriculography (using contrast agents). ${ }^{11}$ The correct diagnosis of this form of HCM is of major importance because many of these patients present at a relatively young age with chest pain and dramatic $T$ wave inversions that frequently result in hospitalization for suspected coronary artery disease. Cardiac catheterization is frequently performed in these patients because of the ECG findings. In our case, coronary angiography was not performed as patient was young and ehocardiographic findings were suggestive of AHC. The electrocardiographic changes in our case were related to $\mathrm{AHC}$.

On 2-D echocardiography, an apical 4-chamber view of the left ventricle revealed hypertrophy of apex, the maximum apical wall thickness was $16 \mathrm{~mm}$. Duygu et al. have shown that mean maximum apical wall thichness was $18 \mathrm{~mm}$ in seventeen patients. ${ }^{7}$ The study of Kitaoka et al. demonstrated that wall thickness at the apex was greater in American patients than in the Japanese patients ( $23 \pm 4$ vs. $18 \pm 2 \mathrm{~mm}$, respectively). ${ }^{12}$

In patients with $\mathrm{AHC}$, a transtorasic echocardiogram may not show the hypertrophy localized to the apex. In adition the apex can be demonstrated by transesophageal echocardiography. 
If echocardiographic images are inadequate, magnetic resonance imaging (MRI) may be used to diagnose this entity. ${ }^{13}$ Sometimes it is confused with apical mural thrombus which can be differentiated by contrast echocardiography of the myocardium. This technique utilizes a contrast agent injected intravenously to highlight hypertrophied myocardium by echocardiography. ${ }^{11}$

As mentioned earlier, AHC is generally considered as having a benign prognosis, one-third of the patients may have severe complications such as myocardial infarction, atrial fibrillation and stroke. ${ }^{7}$ Paroxysmal supraventricular tachycardia and recurrent atrial flutter are also seen in patients with $\mathrm{HCM}^{8}$ Although rare, ventricular tachycardia and sudden death are reported in cases with $\mathrm{AHC} .^{9,} 10$ The life time chance of having myocardial infarction (MI) involving the apical region accounted for $10 \%$ in one study. ${ }^{14}$ Our case also showed a sustained VT

\section{REFERENCES:}

1. Alpendurada F, Prasad SK. The missing spade: apical hypertrophic cardiomyopathy investigation. Int J Cardiovasc Imaging 2008; doi 10.1007/s10554-0089335-z.

2. Moro E, D’angelo G, Nikolosi G.L, Mimo R, Zanuttini D. Long-term evaluation of patients with apical hypertrophic cardiomyopathy. Eur Heart J 1995;16(2):210-7.

3. Sakamoto T, Tei C, Murayama M, Ichiyasu H, Hada Y. Giant $\mathrm{T}$ wave inversion as a manifestation of asymmetrical apical hypertrophy (AAH) of the left ventricle: echocardiographic and ultrasono-cardiotomographic study. Jpn Heart J 1976;17:611-29.

4. Yamaguchi $H$, Ishimura $T$, Nishiyama $S$, Nagasaki F, Nakanishi S, Takatsu F, et al. Hypertrophic nonobstructive cardiomyopathy with giant negative $\mathrm{T}$ waves (apical hypertrophy): ventriculographic and echocardiographic features in 30 patients. Am J Cardiol 1979;44:401-12.

5. Maron BJ. Hypertrophic cardiomyopathy: systematic review. JAMA 2002;287:1308-20.

6. Olearczyk B, Gollol-Raju N, Menzies DJ. Apical hypertrophic cardiomyopathy mimicking acute coronary syndrome: a case report and review of the literature. Angiology 2008;59(5):629-31.

7. Duygu H, Zoghi M, Nalbantgil S, Ozerkan F, Akilli A, Akin $M$, et al. Apical hypertrophic cardiomyopathy might lead to misdiagnosis of ischaemic heart disease. Int J Cardiovasc Imaging 2008; Doi: 10.1007/s10554-0089311-7. that was cardioverted by DC shock. Electrophysiological study with radiofrequency ablation of the re-entrant tract would be the best option for long term prevention of such arrhythmias. Patients are generally kept on beta-blocker attempting to prevent arrhythmias if HCM patients present with arrhythmias of any type unless contra-indicated.

In conclusion, doctors should consider HCM in case of young patients who present with syncope and typical ECG changes. A high index of suspicion with adequate investigations is essential for the proper diagnosis of various variants of HCM. Although echocardiography remains the safe, cheap and non-invasive first choice for investigation, cardiac MRI can be performed. Differentiation of apical hypertrophy from unstable angina and apical thrombus/other masses may be required in certain conditions. Screening of family members is required as this clinical entity has genetic predisposition in most of the times.

8. I.P. Gavaliatsis, N.M. Kouvousis, L.S. Rallidis, et al. Recurrent atrial flutter in apical hypertrophic cardiomyopathy. Jpn Heart J, 33 (1992), pp. 499-504.

9. M.A. Mitchell, S. Nath, K.A. Thompson, et al. Sustained wide complex tachycardia resulting in myocardial injury in a patient with apical hypertrophic cardiomyopathy. Pacing Clin Electrophysiol, 20 (1997), pp. 1866-1869

10. P. Wilson, A. Marks, H. Rastegar, et al. Apical hypertrophic cardiomyopathy presenting with sustained monomorphic ventricular tachycardia and electrocardiographic changes simulating coronary artery disease and left ventricular aneurysm. Clin Cardiol, 13 (1990), pp. 885-887

11. Otieno H, Vivas $\mathrm{Y}$, Traub D. Images in cardiovascular medicine: contrast echocardiography in apical hypertrophic cardiomyopathy. Circulation 2006;11;114(2):e33-4.

12. Kitaoka H, Doi Y, Casey S.A, Hitomi N, Furuno T, Maron B.J. Comparison of prevalence of apical hypertrophic cardiomyopathy in Japan and the United States. Am J Cardiol 2003;92(10):1183-6.

13. Wall EE, Bax JJ, Schalij MJ. Detection of apical hypertrophic cardiomyopathy; which is the appropriate imaging modality. Int J Cardiovasc Imaging 2008; doi:10.1007/ s10554-008-9325-1.

14. A.A. Cubukcu, P.J. Scott, G.J. Williams. Apical hypertrophic cardiomyopathy presenting as acute subendocardial myocardial infarction. Int J Cardiol, 38 (1993), pp. 329-332. 